\title{
Super-luminous Type II supernovae powered by magnetars
}

\author{
Luc Dessart $^{1}$ and Edouard Audit ${ }^{2}$ \\ ${ }^{1}$ Unidad Mixta Internacional Franco-Chilena de Astronomía (CNRS UMI 3386), Departamento de Astronomía, Universidad de \\ Chile, Camino El Observatorio, 1515 Las Condes, Santiago, Chile \\ e-mail: luc.dessart@oca.eu \\ ${ }^{2}$ Maison de la Simulation, CEA, CNRS, Université Paris-Sud, UVSQ, Université Paris-Saclay, 91191 Gif-sur-Yvette, France
}

Received 2 November 2017 / Accepted 18 January 2018

\begin{abstract}
Magnetar power is believed to be at the origin of numerous super-luminous supernovae (SNe) of Type Ic, arising from compact, hydrogen-deficient, Wolf-Rayet type stars. Here, we investigate the properties that magnetar power would have on standard-energy SNe associated with 15-20 $M_{\odot}$ supergiant stars, either red (RSG; extended) or blue (BSG; more compact). We have used a combination of Eulerian gray radiation-hydrodynamics and non-LTE steady-state radiative transfer to study their dynamical, photometric, and spectroscopic properties. Adopting magnetar fields of $1,3.5,7 \times 10^{14} \mathrm{G}$ and rotational energies of $0.4,1$, and $3 \times 10^{51} \mathrm{erg}$, we produce bolometric light curves with a broad maximum covering 50-150 d and a magnitude of $10^{43}-10^{44} \mathrm{erg} \mathrm{s}^{-1}$. The spectra at maximum light are analogous to those of standard SNe II-P but bluer. Although the magnetar energy is channelled in equal proportion between SN kinetic energy and SN luminosity, the latter may be boosted by a factor of 10-100 compared to a standard SN II. This influence breaks the observed relation between brightness and ejecta expansion rate of standard Type II SNe. Magnetar energy injection also delays recombination and may even cause re-ionization, with a reversal in photospheric temperature and velocity. Depositing the magnetar energy in a narrow mass shell at the ejecta base leads to the formation of a dense shell at a few $1000 \mathrm{~km} \mathrm{~s}^{-1}$, which causes a light-curve bump at the end of the photospheric phase. Depositing this energy over a broad range of mass in the inner ejecta, to mimic the effect of multi-dimensional fluid instabilities, prevents the formation of a dense shell and produces an earlier-rising and smoother light curve. The magnetar influence on the SN radiation is generally not visible prior to 20-30 d, during which one may discern a BSG from a RSG progenitor. We propose a magnetar model for the super-luminous Type II SN OGLE-SN14-073.
\end{abstract}

Key words. radiation: dynamics - radiative transfer - supernovae: general - supernova: individual: OGLE-SN2014-073

\section{Introduction}

A large number of super-luminous supernovae (SLSNe) of Type II show unambiguous evidence for interaction with circumstellar material (CSM; Ofek et al. 2007; Smith et al. 2007; Stoll et al. 2011). Because of the large kinetic energy in standard SN ejecta, the observed time-integrated bolometric luminosity can be explained by invoking ejecta deceleration by a massive and dense CSM. The absence of broad, SN-like, lines at early times and the presence of narrow lines over an extended period of time is unambiguous evidence that such an interaction takes place. Some Type II SNe, however, show no obvious signature of interaction despite having a luminosity a factor of 10-100 larger than standard SNe II at maximum, for example, SN 2008es or OGLE-SN14-073 (Gezari et al. 2009; Miller et al. 2009; Terreran et al. 2017). What is striking in these events is the presence of $\mathrm{H}$ I lines well beyond the time of maximum, with a spectral morphology that is analogous to Type II-SNe during the photospheric phase (Gutiérrez et al. 2017). This property excludes a large amount of ${ }^{56} \mathrm{Ni}$ as the power source of the light curve because in that case, the emitting layers at and beyond bolometric maximum are necessarily rich in intermediate mass elements (IMEs) and iron-group elements (IGEs), as obtained in Type II SN models produced by the pair-production instability in super-massive H-rich stars (Dessart et al. 2013b).

An alternative power source to the interaction between ejecta and CSM is the injection of energy from a magnetized fastspinning compact remnant (i.e. a magnetar). Magnetars may be associated with a wide range of astrophysical events, including $\gamma$-ray bursts (Duncan \& Thompson 1992; Usov 1992; Thompson et al. 2004; Bucciantini et al. 2008, 2009; Metzger et al. 2011, 2015), super or even extremely luminous SNe (Kasen \& Bildsten 2010; Woosley 2010; Bersten et al. 2016; Sukhbold \& Woosley 2016), or SNe presenting anomalies in their light curves such as a double bump (Maeda et al. 2007; Taddia et al. 2018). In the context of super-luminous $\mathrm{SNe}$, a fundamental feature of magnetars is their ability to supply power with a large range of magnitudes and time scales without stringent requirements on the ejecta mass or composition. The envelope and core properties of the progenitor might take diverse combinations, in contrast to pair-instability SNe whose large production of ${ }^{56} \mathrm{Ni}$ can only occur in a progenitor of huge mass (Barkat et al. 1967).

Magnetar power has been invoked to explain the doublepeak light curve of SN 2005bf (Maeda et al. 2007; see also Taddia et al. 2018). Kasen \& Bildsten (2010) demonstrated that some combination of magnetar field strength and initial spin period (as well as ejecta mass and kinetic energy) could explain the observations of the Type II SN 2008es (Gezari et al. 2009; Miller et al. 2009) and the type Ic SN 2007bi (Gal-Yam et al. 2009). More recently, a large number of Type Ic SLSNe have been discovered and followed photometrically and spectroscopically from early to late times. Their radiative properties favor a magnetar origin in a massive Wolf-Rayet progenitor (Inserra et al. 2013; Nicholl et al. 2013, 2014; Jerkstrand et al. 2017). In most of these SLSNe Ic, distinguishing between ${ }^{56} \mathrm{Ni}$ power and magnetar power can in fact be done from a single spectrum 
taken around bolometric maximum (Dessart et al. 2012). While most simulations have assumed spherical symmetry, a few radiation-hydrodynamics simulations performed in two dimensions suggest the occurrence of strong Rayleigh-Taylor driven mixing in the inner ejecta, affecting the thermal and density structures of a large fraction of the ejecta, which may affect the emergent radiation (Chen et al. 2016; Suzuki \& Maeda 2017).

In this paper, we present results from numerical simulations of magnetar powered $\mathrm{SNe}$ resulting from the explosion of a red-supergiant (RSG) or a blue-supergiant (BSG) star. In the next section, we present the numerical setup for the radiation hydrodynamics and for the radiative transfer calculations. We then present our results. In Sect. 3, we first discuss the influence of the adopted energy-deposition profile. We then describe the impact of the chemical stratification on the bolometric light curve (Sect. 4). In Sect. 5, we present the light curve differences obtained for the explosion of a BSG and a RSG progenitor influenced by the same magnetar. Using a given explosion model from a RSG progenitor, we explore the diversity of light curves and ejecta properties resulting from a grid of magnetar fields and spin periods (Sect. 6). In Sect. 7, we discuss our model results in the context of observations. We present our conclusions in Sect. 8 .

\section{Numerical setup}

We used the Eulerian radiation hydrodynamics code HERACLES (González et al. 2007; Vaytet et al. 2011) to simulate the influence of magnetar power on a small set of SN ejecta models. We first describe the code assumptions and set up, then the treatment of magnetar power, followed by the progenitor models employed, and finally the post-processing for spectral calculations. A summary of properties from our simulations (initial conditions and results) is given in Table 1.

\subsection{Numerical approach with HERACLES}

For the radiation-hydrodynamics simulations with HERACLES we assume spherical symmetry. This choice is most likely inadequate to describe the complex geometry of this system, both on large scales (see, e.g., Burrows et al. 2007; Bucciantini et al. 2009; Mösta et al. 2015) and small scales (Chen et al. 2016; Suzuki \& Maeda 2017). Spherical symmetry has been assumed so far in all simulations of magnetar-powered SN light curves and we make the same simplification for convenience for the time being. We also employ a gray approximation for the radiative transfer (one energy/frequency group). In the 1D simulations of interacting SNe presented in Dessart et al. (2015, 2016), we used a multi-group approach because the radiation and the gas could be strongly out of equilibrium, which does not apply as much here. We adopt a simple equation of state that treats the gas as ideal with $\gamma=5 / 3$ and a mean atomic weight $\bar{A}$ of 1.35 . The thermal energy of the gas is a tiny fraction of the radiative energy so the neglect, for example, of changes in the level of excitation and ionization of the gas has little impact on either the radiative or dynamical properties. The changes in ionization are accounted for in the computation of the Rossland mean opacity so the electron scattering opacity, which is the main contribution to the total opacity, is accurately accounted for. The code distinguishes absorptive from scattering opacity. At each grid point, the absorptive opacity is obtained by subtracting the electron scattering contribution to the Rosseland mean opacity (see Dessart et al. 2015 for details on how we compute these opacities). We have opacity tables for up to five different compositions, characteristic of a massive star envelope at core collapse.
Namely, we adopt the composition of the H-rich envelope, the He-rich shell, the O-rich shell, the Si-rich shell, and a shell dominated by IGEs. For simplicity, most of the simulations presented here adopt a composition with $X_{\mathrm{H}}=0.65, X_{\mathrm{He}}=0.33$, and a solar composition for heavy elements (again, this composition is only relevant for the computation of the opacities).

To better resolve the ejecta at smaller radii, we use a grid with a constant spacing from the minimum radius at $\sim 10^{13} \mathrm{~cm}$ up to $R_{\mathrm{t}}=5 \times 10^{14} \mathrm{~cm}$, and then switch to a grid with a constant spacing in the log up to $10^{16} \mathrm{~cm}$. The grid is designed to have no sharp jump in spacing at $R_{\mathrm{t}}$. For the boundary conditions, we adopt for both the gas and the radiation a reflecting inner boundary and a free-flow outer boundary. The bolometric light curves that we extract from the HERACLES simulations are computed using the total radiative flux at the outer boundary. The light travel time to the outer boundary at $10^{16} \mathrm{~cm}$ introduces a delay for the record of the emergent radiation, by at most 3-4d.

Radioactive decay is ignored in the present work.

\subsection{Treatment of magnetar energy deposition}

We use the formulation of Kasen \& Bildsten (2010) for the magnetar power as a function of time since magnetar birth:

$\dot{e}_{\mathrm{pm}}=\left(E_{\mathrm{pm}} / t_{\mathrm{pm}}\right) /\left(1+\left(t+\delta t_{\mathrm{pm}}\right) / t_{\mathrm{pm}}\right)^{2}$

with

$t_{\mathrm{pm}}=\frac{6 I_{\mathrm{pm}} c^{3}}{B_{\mathrm{pm}}^{2} R_{\mathrm{pm}}^{6} \omega_{\mathrm{pm}}^{2}}$,

and where $E_{\mathrm{pm}}, B_{\mathrm{pm}}, R_{\mathrm{pm}}, I_{\mathrm{pm}}$ and $\omega_{\mathrm{pm}}$ are the initial rotational energy, magnetic field, radius, moment of inertia, and angular velocity of the magnetar; $\delta t_{\mathrm{pm}}$ is the elapsed time since magnetar birth at the start of the HERACLES simulation; and $c$ is the speed of light. We adopt $I_{\mathrm{pm}}=10^{45} \mathrm{~g} \mathrm{~cm}^{2}$ and $R_{\mathrm{pm}}=10^{6} \mathrm{~cm}$. We use here the special case of a magnetic dipole spin down. At late times, the magnetar power scales as $1 /\left(B^{2} t^{2}\right)$.

The term $\delta t_{\mathrm{pm}}$ is about $10^{5} \mathrm{~s}$ in our HERACLES simulations because we do not start at the time of explosion. Instead, we start from an already existing ejecta at about $1 \mathrm{~d}$ after magnetar birth. This corresponds to a time shortly before shock breakout in our RSG progenitor, and well after shock breakout in out BSG model (see next section).

In our simulations, we ignore the magnetar energy deposited during the first $10^{5} \mathrm{~s}$ that follow core bounce and the magnetar birth. This energy corresponds to

$E_{\text {neglected }}=E_{\mathrm{pm}}\left(1-1 /\left(1+\delta t_{\mathrm{pm}} / t_{\mathrm{pm}}\right)\right)$.

$E_{\text {neglected }}$ increases with $E_{\mathrm{pm}}$ and $B_{\mathrm{pm}}$. For example, it can be as much as $1.8 \times 10^{51} \mathrm{erg}$ for $E_{\mathrm{pm}}=3 \times 10^{51} \mathrm{erg}$ and $B_{\mathrm{pm}}=7 \times 10^{14} \mathrm{G}$ (so about $60 \%$ of $E_{\mathrm{pm}}$ ), while it is merely $1.7 \times 10^{48} \mathrm{erg}$ for $E_{\mathrm{pm}}=0.4 \times 10^{51} \mathrm{erg}$ and $B_{\mathrm{pm}}=10^{14} \mathrm{G}$ (so less than a per cent of $E_{\mathrm{pm}}$ ). This neglect impacts moderately the emergent radiation because the energy deposited prior to one day is mostly used to boost the ejecta kinetic energy while it is strongly degraded by ejecta expansion.

The energy-deposition scheme is presented in detail in Sect. 3.

\subsection{Progenitor models and initial conditions for the HERACLES simulations}

We use two different models for the initial conditions in HERACLES. The first model (m15mlt3 of Dessart et al. 2013a) 
Table 1. Summary of model properties for the progenitor, the ejecta, the magnetar, and some results at bolometric maximum obtained with the HERACLES simulations.

\begin{tabular}{lccccccccccc}
\hline \hline Model & $\begin{array}{c}R_{\star} \\
{\left[R_{\odot}\right]}\end{array}$ & $\begin{array}{c}M_{\mathrm{ej}} \\
{\left[M_{\odot}\right]}\end{array}$ & $\begin{array}{c}E_{\mathrm{kin}} \\
{[\mathrm{erg}]}\end{array}$ & $\begin{array}{c}E_{\mathrm{pm}} \\
{[\mathrm{erg}]}\end{array}$ & $\begin{array}{c}B_{\mathrm{pm}} \\
{[\mathrm{G}]}\end{array}$ & $\begin{array}{c}m_{\mathrm{lim}} \\
{\left[M_{\odot}\right]}\end{array}$ & $\begin{array}{c}d m_{\text {lim }} \\
{\left[M_{\odot}\right]}\end{array}$ & $\begin{array}{c}t_{\mathrm{pm}} \\
{[\mathrm{d}]}\end{array}$ & $\begin{array}{c}t_{\text {peak }} \\
{[\mathrm{d}]}\end{array}$ & $\begin{array}{c}L_{\text {peak }} \\
{\left[\mathrm{erg} \mathrm{s}^{-1}\right]}\end{array}$ & $\begin{array}{c}V_{\text {phot,peak }} \\
{\left[\mathrm{km} \mathrm{s}^{-1}\right]}\end{array}$ \\
\hline RE0p4B3p5 & 501 & 11.9 & $0.92(51)$ & $0.4(51)$ & $3.5(14)$ & 1.0 & 4.0 & 19 & 99 & $1.10(43)$ & 3540 \\
RE0p4B3p5d & 501 & 11.9 & $0.92(51)$ & $0.4(51)$ & $3.5(14)$ & 0.1 & 0.0 & 19 & 160 & $1.14(43)$ & 2560 \\
RE0p4B3p5x & 501 & 11.9 & $0.92(51)$ & $0.4(51)$ & $3.5(14)$ & 1.0 & 4.0 & 19 & 102 & $1.14(43)$ & 3510 \\
\hline RE0p4B1 & 501 & 11.9 & $0.92(51)$ & $0.4(51)$ & $1.0(14)$ & 1.0 & 4.0 & 234 & 126 & $9.46(42)$ & 2960 \\
RE0p4B3p5 & 501 & 11.9 & $0.92(51)$ & $0.4(51)$ & $3.5(14)$ & 1.0 & 4.0 & 19 & 99 & $1.10(43)$ & 3540 \\
RE0p4B7 & 501 & 11.9 & $0.92(51)$ & $0.4(51)$ & $7.0(14)$ & 1.0 & 4.0 & 5 & 86 & $7.06(42)$ & 3400 \\
RE1B1 & 501 & 11.9 & $0.92(51)$ & $1.0(51)$ & $1.0(14)$ & 1.0 & 4.0 & 94 & 125 & $2.84(43)$ & 4030 \\
RE1B3p5 & 501 & 11.9 & $0.92(51)$ & $1.0(51)$ & $3.5(14)$ & 1.0 & 4.0 & 8 & 76 & $1.77(43)$ & 4880 \\
RE1B7 & 501 & 11.9 & $0.92(51)$ & $1.0(51)$ & $7.0(14)$ & 1.0 & 4.0 & 2 & 89 & $9.30(42)$ & 3820 \\
RE3B1 & 501 & 11.9 & $0.92(51)$ & $3.0(51)$ & $1.0(14)$ & 1.0 & 4.0 & 31 & 86 & $7.78(43)$ & 5680 \\
RE3B3p5 & 501 & 11.9 & $0.92(51)$ & $3.0(51)$ & $3.5(14)$ & 1.0 & 4.0 & 2.6 & 51 & $2.86(43)$ & 6200 \\
RE3B7 & 501 & 11.9 & $0.92(51)$ & $3.0(51)$ & $7.0(14)$ & 1.0 & 4.0 & 0.6 & 90 & $1.19(43)$ & 4390 \\
\hline RE0p4B3p5 & 501 & 11.9 & $0.92(51)$ & $0.4(51)$ & $3.5(14)$ & 1.0 & 4.0 & 19 & 99 & $1.10(43)$ & 3540 \\
BE0p4B3p5 & 47 & 15.6 & $1.20(51)$ & $0.4(51)$ & $3.5(14)$ & 1.0 & 4.0 & 19 & 105 & $9.57(42)$ & 3230 \\
\hline RE0p4B4p50 & 501 & 11.9 & $0.92(51)$ & $0.4(51)$ & $4.5(14)$ & 2.5 & 5.0 & 12 & 85 & $9.62(42)$ & 4060 \\
RE0p4B4p5os & 501 & 17.8 & $2.67(51)$ & $0.4(51)$ & $4.5(14)$ & 2.5 & 5.0 & 12 & 83 & $1.09(43)$ & 4280 \\
\hline
\end{tabular}

derives from a red-supergiant (RSG) model of a $15 M_{\odot}$ star on the main sequence. At core collapse, it corresponds to a star with a total mass of $14.1 M_{\odot}$, a surface radius of $500 R_{\odot}$, a luminosity of $64200 L_{\odot}$. For the corresponding explosion, the total ejecta mass is $11.9 M_{\odot}$ and the explosion energy is $0.92 \times 10^{51} \mathrm{erg}$ (slightly different from the value in Dessart et al. 2013a because of remapping on a lower resolution Eulerian grid initially and trimming of the inner regions at small radii). The ${ }^{56} \mathrm{Ni}$ yield is irrelevant since radioactive decay is ignored in this study. The second model ( $\operatorname{lm} 18 \mathrm{a} 7 \mathrm{Ad}$ of Dessart \& Hillier 2010) derives from a BSG model of a $18 M_{\odot}$ star on the main sequence (evolved at a metallicity of 0.008 rather than solar). At the onset of collapse, it corresponds to a star with a total mass of $17 M_{\odot}$, a surface radius of $47 R_{\odot}$, and a luminosity of $210000 L_{\odot}$. For the corresponding explosion, the total ejecta mass is $15.6 M_{\odot}$ and the explosion energy is $1.20 \times 10^{51} \mathrm{erg}$. Without magnetar power, these models reproduce closely the observed properties of the standard Type II-P SN 1999em and the Type II-peculiar SN 1987A (see Dessart et al. 2013a; Dessart \& Hillier 2010 for discussion and results).

These two models are used to cover the range of radii for Type II supernova progenitors, since the envelope extent is the fundamental characteristic that distinguishes the progenitors of Type II-Plateau and Type II-Peculiar SNe. Different progenitor main sequence masses and/or adopted wind mass loss rates would also impact the resulting observables of our magnetarpowered Type II SNe. This second aspect is left to a future study.

Because the HERACLES code is Eulerian, we start from an already existing ejecta rather than one at core bounce. For the RSG progenitor, we take the model at $10^{5} \mathrm{~s}$ after the explosion trigger, which corresponds to about one hour before shock breakout. For the BSG progenitor we take the same starting time, but because of the reduced progenitor radius (i.e., $50 R_{\odot}$ ), this time is well after shock breakout. Since we focus on relatively massive ejecta for which the rise time to bolometric maximum (excluding the initial shock breakout burst) is weeks to months, this initial offset has little impact (see also discussion in the previous section).
We also need to specify the conditions between the outer edge of the progenitor/ejecta at $10^{5} \mathrm{~s}$ and the outer grid radius at $10^{16} \mathrm{~cm}$. For simplicity, we fill this volume with a lowdensity low-temperature (set to $2000 \mathrm{~K}$ ) material, reflecting a stellar wind mass loss rate of $10^{-6} M_{\odot} \mathrm{yr}^{-1}$. A constant wind velocity of $50 \mathrm{~km} \mathrm{~s}^{-1}$ is used for the RSG progenitor model, and $500 \mathrm{~km} \mathrm{~s}^{-1}$ for the BSG progenitor model. ${ }^{1}$ These wind velocities are approximate but have little impact on the results discussed here. Indeed, the ejecta/wind interaction contributes a few percent of the total bolometric luminosity. This contribution persists until the ejecta/wind interaction crosses the outer boundary of the Eulerian grid, at $125 \mathrm{~d}(50 \mathrm{~d})$ in the simulations based on the RSG (BSG) progenitor.

Our model nomenclature is to use prefix " $R$ " ("B") to refer to simulations based on the RSG (BSG) models. We then append this prefix by values for the magnetar initial rotational energy and magnetic field. For example, simulation RE1B3p5 employs the RSG progenitor model, a magnetar initial rotational energy of $10^{51} \mathrm{erg}$ and a magnetic field strength of $3.5 \times 10^{14} \mathrm{G}$. In Sects. 3 and 4 , we explore special cases for which we stitch an additional suffix (for example "x" or "d").

\subsection{Spectral simulations}

We post-process the HERACLES simulations at the time when the SN reaches bolometric maximum, which is around $100 \mathrm{~d}$ after the start of the simulation for our sample. We use CMFGEN in a steady-state mode and adopt the ejecta properties from HERACLES (i.e., radius, velocity, density, temperature). Homologous expansion is not assumed, which is why we read both the radius and the velocity. In most simulations, we adopt a uniform composition of a RSG star at death. We use mass fractions of 0.66 for $\mathrm{H}, 0.32$ for $\mathrm{He}, 0.0019$ for $\mathrm{C}, 0.004$ for $\mathrm{N}, 0.008$ for $\mathrm{O}$, and use the solar metallicity value for heavier elements. The CMFGEN simulations treat $\mathrm{H}, \mathrm{He}, \mathrm{C}, \mathrm{N}, \mathrm{O}, \mathrm{Na}, \mathrm{Si}, \mathrm{Ca}, \mathrm{Ti}, \mathrm{Sc}$, and Fe. The model atom includes H I, He I- II, C I- III, N I-III,

\footnotetext{
1 Our BSG progenitor/explosion model is suitable for SN 1987A but the adopted wind mass loss rate is too large and thus not strictly appropriate (see e.g. Chevalier \& Dwarkadas 1995).
} 

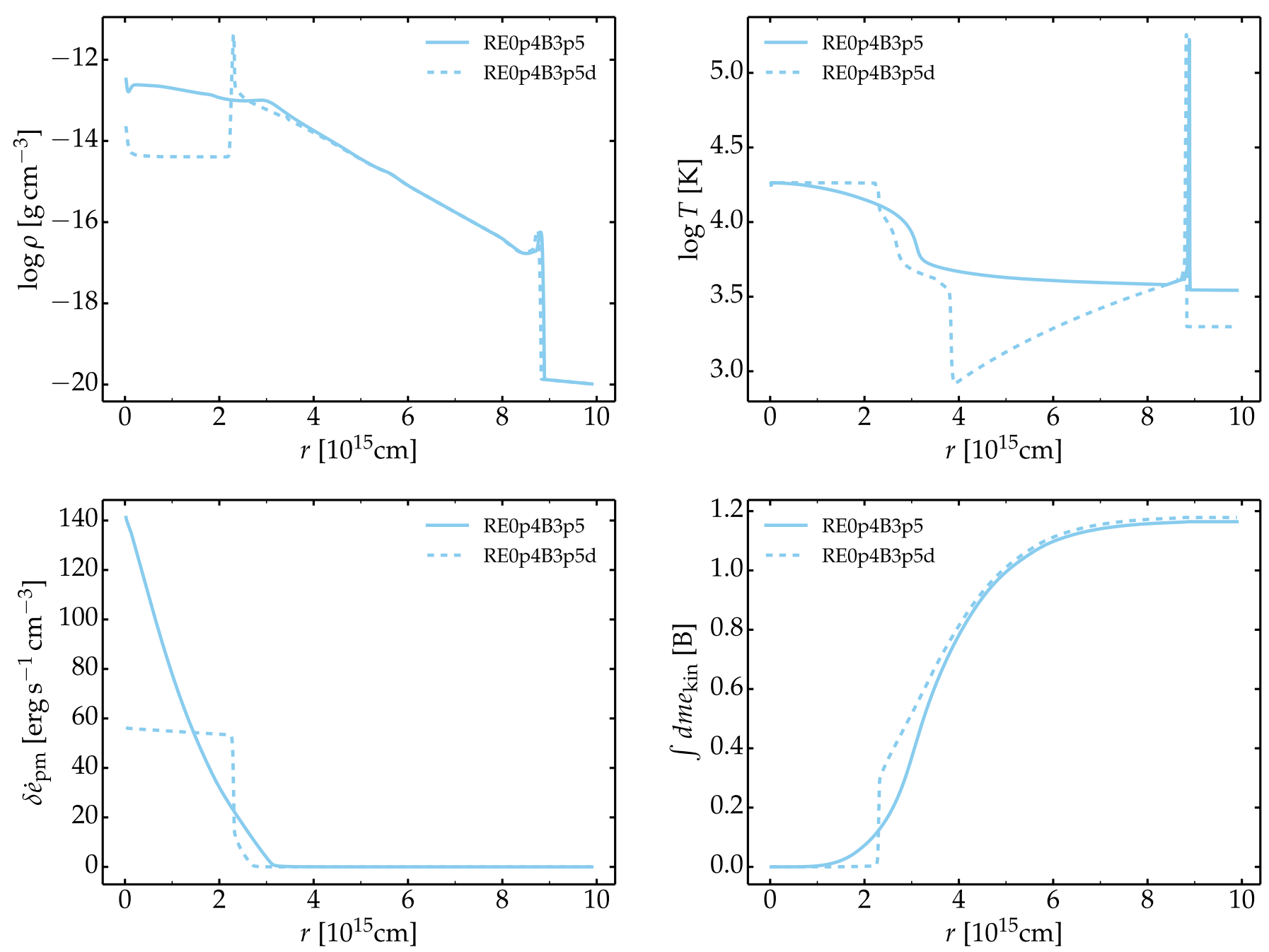

Fig. 1. Comparison of models RE0p4B3p5 and RE0p4B3p5d to test the influence of the radial profile used for the deposition of magnetar power. Ordered clockwise from top left, we show the radial variation of the mass density, temperature, local emissivity from magnetar power, and the cumulative kinetic energy integrated outward from the inner boundary, all at 116 d after the start of the HERACLES simulation. Model RE0p4B3p5 spreads the magnetar energy over a large range of masses, while model RE0p4B3p5d deposits all the magnetar energy over the inner $0.1 M_{\odot}$.

O I- III, Na I, Si II-III, Ca II, Ti II, Sc II, and Fe I-IV. For the starting level populations in CMFGEN, we adopt local thermodynamic equilibrium (LTE) and then evolve the non-LTE solution with the temperature fixed to what it was in HERACLES.

Evolving CMFGEN at a fixed temperature is not consistent but it provides a useful estimate of the emergent spectral energy distribution as well as the ionization, color, and line profile width/strength. During the photospheric phase, the ejecta is optically thick and lines do not dominate the luminosity. At late times, when the ejecta is optically thin, lines become the main coolant so that the LTE conditions assumed for the gas state in HERACLES are no longer suitable for a post-processing with CMFGEN. The CMFGEN simulations presented here are therefore limited to the photospheric phase. We use the (unambiguous) time of maximum for our comparisons.

\section{Influence of energy deposition profile}

We first start with the problematic issue of the treatment of magnetar energy deposition. The newly-born hot magnetar probably emits high energy photons in the X-ray and $\gamma$-ray range as well as leptons (electrons and positrons). The lepton energy should be efficiently thermalized but high energy photons have a much larger mean free path and may eventually deposit their energy far from their production site (they may even escape).

Secondly, as expected and demonstrated in recent 2D simulations (Chen et al. 2016; Suzuki \& Maeda 2017), even if one assumes that the energy is deposited and thermalized at the ejecta base, this magnetar energy injection gives rise to strong Rayleigh-Taylor mixing in the inner ejecta. Rather than slowly diffusing out through the ejecta as it expands, the magnetar energy is more rapidly advected out by turbulent motions. In multiple dimensions, this turbulence prevents the formation of a fast moving dense shell in the inner ejecta, whose occurrence is an artifact of assuming spherical symmetry (Kasen \& Bildsten 2010; Woosley 2010).

In this work, to mimic the effects of multi-dimensional instabilities seen in the simulations of Chen et al. (2016) and Suzuki \& Maeda (2017), as well as the non-local nature of energy deposition of the magnetar, we deposit the magnetar energy over a range of mass shells rather than at the base of the ejecta. We use an energy deposition that has the same profile as the density, with an additional weight set to unity below a certain mass limit $m_{\text {lim. }}$. In ejecta mass shells $m$ beyond $m_{\text {lim }}$, the weight is either set to zero or to $\exp \left(-x^{2}\right)$, where $x=(m-$ $\left.m_{\text {lim }}\right) / d m_{\lim }$ ( $m$ is taken as zero at the ejecta base). The adopted energy deposition profile is fixed in mass space throughout the 
simulation. This is clearly very simplistic but it will allow us to gauge the impact on observables. In the future, it will be desirable to improve on this by performing multi-group radiative transfer (to solve for the transport of both high energy and low-energy photons), coupled with multi-dimensional hydrodynamics to describe adequately the contributions of energy transport by advection, diffusion, and non-local energy deposition (from high energy particles and photons with a large mean free path).

To test the influence of the magnetar energy deposition profile, we have run two simulations based on the RSG progenitor model and influenced by a magnetar with an initial rotational energy of $0.4 \times 10^{51} \mathrm{erg}$ and a magnetar field strength of $3.5 \times 10^{14} \mathrm{G}$. In model RE0p4B3p5d, the weight is set to unity below $m_{\lim }=0.1 M_{\odot}$, and to zero above, that is the energy is deposited over a narrow mass range of $0.1 M_{\odot}$ above the ejecta base. In model RE0p4B3p5, the weight is set to unity below $m_{\text {lim }}=1.0 M_{\odot}$, and to $\exp \left(-x^{2}\right)$ above, with $d m_{\lim }=4.0 M_{\odot}$. The corresponding volume integrated emissivity is then normalized to the magnetar power at the time.

We show a set of results for this test comparison in Fig. 1, including the density, the temperature, the energy-deposition profile, and the cumulative kinetic energy versus radius (the time is $116 \mathrm{~d}$, or $10^{7} \mathrm{~s}$, for each simulation). In the case where the magnetar energy is deposited in the inner $0.1 M_{\odot}$ of the ejecta, the density profile exhibits a dense shell at $\sim 2.2 \times 10^{15} \mathrm{~cm}$ and $2200 \mathrm{~km} \mathrm{~s}^{-1}$ (the velocity profile, not shown, is essentially homologous for each simulation), with little mass below it. The temperature profile shows much more structure, with sharp variations in the cool regions above the photosphere. The temperature spike at large radii in both simulations corresponds to the shock with the surrounding low-density wind - the interaction power is small in comparison to the magnetar power.

The impact on the light curve is significant (Fig. 2). The time-integrated luminosity is greater by $1.5 \times 10^{49} \mathrm{erg}$ in the model RE0p4B3p5 characterized by a smooth/extended magnetar energy deposition. This excess radiative energy in model RE0p4B3p5 yields instead an excess kinetic energy in the model RE0p4B3p5d. This is a one per cent difference since the total ejecta kinetic energy is $\leqslant 1.2 \times 10^{51}$ erg (bottom right panel in Fig. 1). The morphology of the light curve is also affected by the adopted treatment of magnetar energy deposition. Model RE0p4B3p5d, in which the deposition is confined to the innermost ejecta layers and causes the formation of a dense shell, the light curve shows a pronounced bump at $150 \mathrm{~d}$, which corresponds to the epoch when the photosphere recedes to those deep ejecta layers. Model RE0p4B3p5, in which the deposition is spread in mass space shows a smooth bolometric light curve. In this case, the onset of brightening also occurs $\sim 20 \mathrm{~d}$ sooner because of the energy deposition further out in the ejecta, at smaller optical depths. The simulations of Kasen \& Bildsten (2010) do not show any jump in the light curve despite the formation of a dense shell at the base of their ejecta. This is probably because they adopt a fixed opacity, independent of ionization. Our models are $\mathrm{H}$ rich and the opacity varies steeply when $\mathrm{H}$ recombines, as occurs at $150 \mathrm{~d}$ in the dense shell formed in model RE0p4B3p5d.

While the energy deposition implemented in both models is artificial, the smooth and extended deposition profile adopted for model RE0p4B3p5 yields ejecta properties in better agreement with the 2-D simulations of Chen et al. (2016) and Suzuki \& Maeda (2017), in particular with the lack of a dense shell in the inner ejecta. A similar effect on the density structure and on the resulting SN light curve is seen in radiation-hydrodynamics

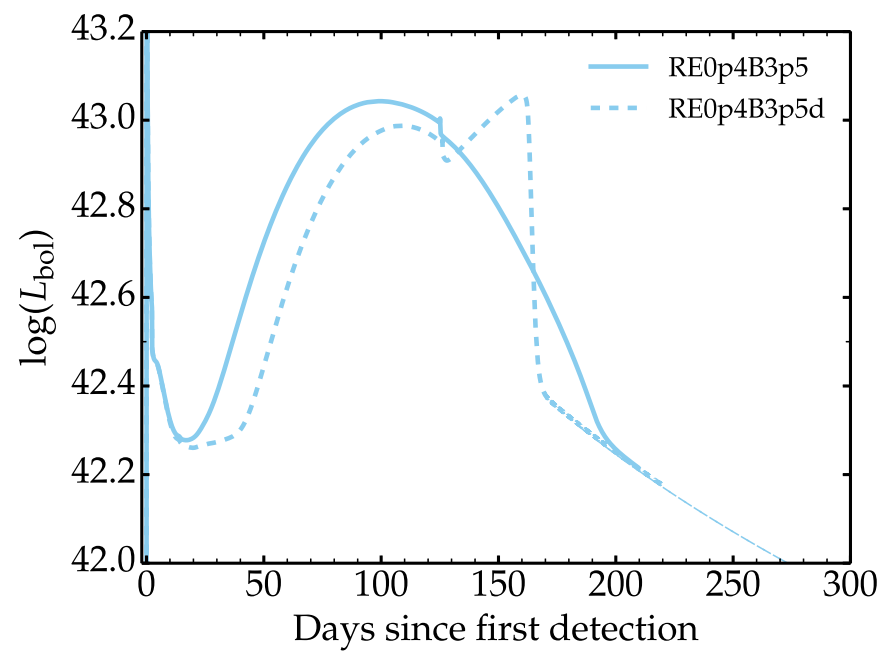

Fig. 2. Comparison of the bolometric light curve for simulations RE0p4B3p5 and RE0p4B3p5d, in which only the magnetar energy deposition profile differs (see Fig. 1, and Sect. 3 for discussion). The glitch at $125 \mathrm{~d}$ occurs when the outer shock with the progenitor wind leaves the Eulerian grid at $10^{16} \mathrm{~cm}$. The broad bump at $150-170 \mathrm{~d}$ for model RE0p4B3p5d occurs when the photosphere reaches the dense shell in the inner ejecta; the sharp drop at $170 \mathrm{~d}$ is when this dense shell becomes optically thin.

simulations based on 3D simulations of core-collapse $\mathrm{SNe}$ (Utrobin et al. 2017).

In the remaining of this work, we employ the same parametrized energy deposition profile as for RE0p4B3p5. Our magnetar-powered simulations will therefore tend to produce material distributed smoothly in velocity space down to very small values.

\section{Influence of chemical stratification}

We also tested the influence of chemical stratification on our results with HERACLES. In HER ACLES, one can follow the evolution of "scalars" from their initial distribution. In our Type II SN explosions, we approximate the ejecta composition using only the five most important species. We focus on the distribution of $\mathrm{H}, \mathrm{He}, \mathrm{O}, \mathrm{Si}$, and the initial ${ }^{56} \mathrm{Ni}$ (here, ${ }^{56} \mathrm{Ni}$ is just a tracer for IGEs since we ignore radioactive decay). We renormalize the mass fraction to unity to correct for the missing species. The only impact of this chemical stratification on the numerical setup is through the opacity tables. Rather than using one table for an H-rich composition, we now use five tables to track the evolution in mean atomic weight $\bar{A}$ from the outer layers rich in $\mathrm{H}$ and $\mathrm{He}(\bar{A} \approx 1.35)$ down to the $\mathrm{O}-\mathrm{Si}$ rich layers $(\bar{A} \approx 17.0$; this value depends on the progenitor/ejecta composition and the adopted mixing). We use the Rosseland mean opacity in our calculations so we account for the continuum opacity from bound-free and free-free processes, and from electron scattering opacity, as well as line opacity.

We have tested the impact of chemical stratification using model RE0p4B3p5x - model RE0p4B3p5 has a uniform composition representative of the progenitor surface. We find no difference in dynamical properties between model RE0p4B3p5x and model RE0p4B3p5. The gas density matters but not the precise distribution of this mass between different species/isotopes. Since we use the same equation of state for both models, we neglect the change in energy release from recombination and de-excitation of atoms/ions. However, the thermal pressure is 
always a tiny fraction of the total pressure in $\mathrm{SNe}$, and even more so in magnetar-powered SNe.

Figure 3 shows the bolometric light curves for models RE0p4B3p5x and RE0p4B3p5. The difference is negligible at early times, which is expected since more than half the ejecta is made of the progenitor $\mathrm{H}$-rich envelope. At and after maximum, a small difference is seen, RE0p4B3p5x being first brighter and then fainter than model RE0p4B3p5. We interpret this results as arising from the earlier release of trapped radiation energy in model RE0p4B3p5x. The greater abundance of heavy elements in model RE0p4B3p5x leads to a (modest) reduction in opacity, which makes the trapping of radiation less efficient. We use the Rosseland mean in our calculations so the reduction in opacity is driven by the reduction in electron-scattering opacity, and is not compensated by the greater metal line opacity at large $\bar{A}$.

\section{Influence of the initial structure and radius: BSG versus RSG progenitors}

Kasen \& Bildsten (2010) considered a variety of magnetar properties (magnetic field and initial rotational energy), ejecta masses, and ejecta kinetic energies. However, they neglect the initial internal energy left by the shock passage (and that does not end up as kinetic energy for the ejecta), since this energy is typically much less than the energy released by a magnetar in a SLSN. One consequence in their simulation is a low predicted luminosity at times shorter than about the magnetar spin down time scale. In reality, during this early phase, an important source of energy is the shock deposited energy - it is even the dominant source of energy in the explosion of supergiant stars. Indeed, in supergiant stars, this energy is large, not strongly degraded by expansion, and allows even a standard SN II-P to radiate at $10^{9} L_{\odot}$ for many days after the shock emergence. Accounting for the large size of supergiant progenitors is therefore necessary to produce a consistent light curve prior to maximum (although the magnetar energy deposition may extend far out in the ejecta and affect the SN brightness very soon after explosion).

Here, we compare the predictions for the influence of a magnetar $\left(E_{\mathrm{pm}}=0.4 \times 10^{51} \mathrm{erg}, B_{\mathrm{pm}}=3.5 \times 10^{14} \mathrm{G}\right)$ acting on a SN ejecta that resulted from the explosion of a BSG and a RSG progenitor star. As discussed in Sect. 2.3, these models yield properties similar to SN 1987A and SN 1999em when evolved with no magnetar influence (Dessart \& Hillier 2010; Dessart et al. 2013a).

The left panel in Fig. 4 presents the bolometric light curve of models BE0p4B3p5 and RE0p4B3p5. The thick dashed lines correspond to the resulting HERACLES light curve with no magnetar and no ${ }^{56} \mathrm{Ni}$ (we ignore radioactive decay in this study). For up to $20-30 \mathrm{~d}$, the model luminosity is not influenced by the magnetar in our setup, but beyond that, the light curves of models BE0p4B3p5 and RE0p4B3p5 slowly converge and eventually overlap soon after maximum. The resulting light curves have a bell shape morphology. As expected, the original star size matters only at early times, while at late times, the power supply is so large that it overwhelms the slight differences that the two models may have had at explosion or at collapse. But the sizable differences at early times may allow to constrain the progenitor size, in the same fashion as for distinguishing SNe II-pec from SNe II-P (e.g., SN 1987A from SN 1999em, for which the differences at early times are well documented).

The right panel in Fig. 4 shows the optical spectra for each model around the time of bolometric maximum. The differences

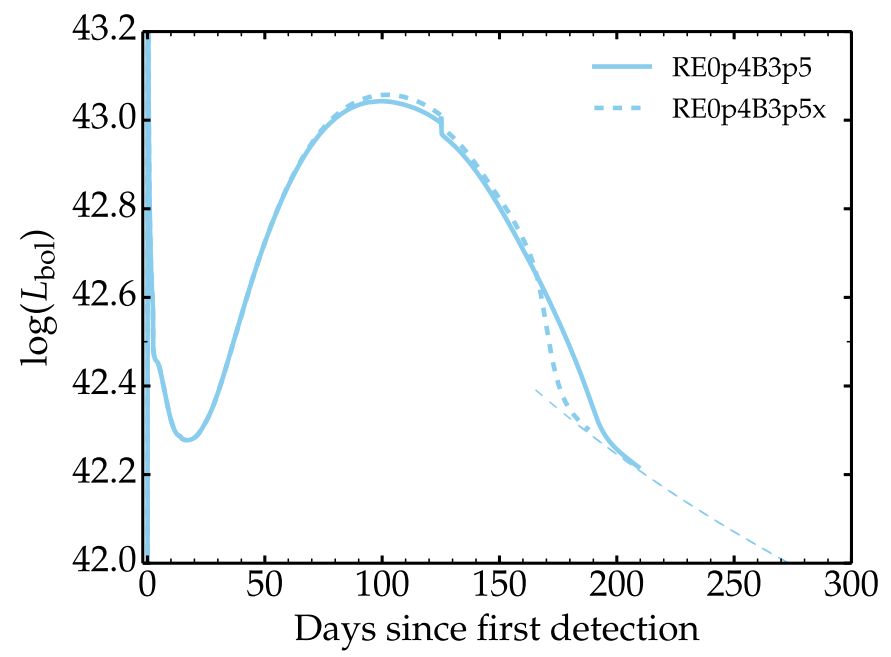

Fig. 3. Bolometric light curves computed with HERACLES for models RE0p4B3p5 (homogenous composition with $\bar{A}=1.35$ ) and RE0p4B3p5x (chemically stratified and spanning $\bar{A}=1.35$ up to 17). Allowing for a depth dependent abundance only affects the light curve after maximum, when the former (metal-rich) He-core material is progressively revealed. The impact on the light curve is however minor here. The glitch at $125 \mathrm{~d}$ occurs when the outer shock with the progenitor wind leaves the Eulerian grid at $10^{16} \mathrm{~cm}$. The spectra for these two models at maximum light are identical (hence not shown). This is because the spectrum then forms within the $\mathrm{H}$-rich layers, and thus at the same composition in both models.

are marginal. It is important to notice that in such magnetarpowered SNe from H-rich stars, the spectra at maximum light show a typical Type II spectrum. In a model whose bolometric maximum is powered by the decay from a large mass of ${ }^{56} \mathrm{Ni}$, the optical spectra at bolometric maximum tend to show lines from IMEs, although $\mathrm{H}$ I lines may still be seen if the progenitor radius is huge (Dessart et al. 2013b).

\section{Results for a grid of RSG-star explosions influenced by magnetar power}

In this section, we present results for a grid of simulations in which the magnetar properties are varied. Using the RSG progenitor model, we cover magnetar initial rotational energies of $0.4,1$, and $3.5 \times 10^{51} \mathrm{erg}$ (corresponding for our adopted neutron star to initial spin periods of 7.0, 4.4, and $2.6 \mathrm{~ms}$ ) and magnetic field strength of $1,3.5$, and $7 \times 10^{14} \mathrm{G}$. The magnetar spin down timescale covers from a day to several months (Table 1; $t_{\mathrm{pm}}$ scales as the inverse of $\left.B_{\mathrm{pm}}^{2} E_{\mathrm{pm}}\right)$.

\subsection{Bolometric light curves}

We show the bolometric light curves for this set of models in Fig. 5. The light curves all start at the same level, when the magnetar influence has not yet been felt in the outer ejecta. This influence occurs sooner for a shorter spin down timescale $t_{\mathrm{pm}}$. This depends also on the magnetar energy $E_{\mathrm{pm}}$ in our simulations but quantitatively, we need to be cautious since the energy released prior to $1 \mathrm{~d}$ is not accounted for in our simulations (this impacts our results for small values of $t_{\mathrm{pm}}$ ). The earlier the bolometric light curve rises again, the faster is the rise to the bolometric maximum, which occurs in our set of simulations between 50 and $125 \mathrm{~d}$ after explosion. The bolometric maximum spans the range $0.7-7.8 \times 10^{43} \mathrm{erg} \mathrm{s}^{-1}$. These values for 

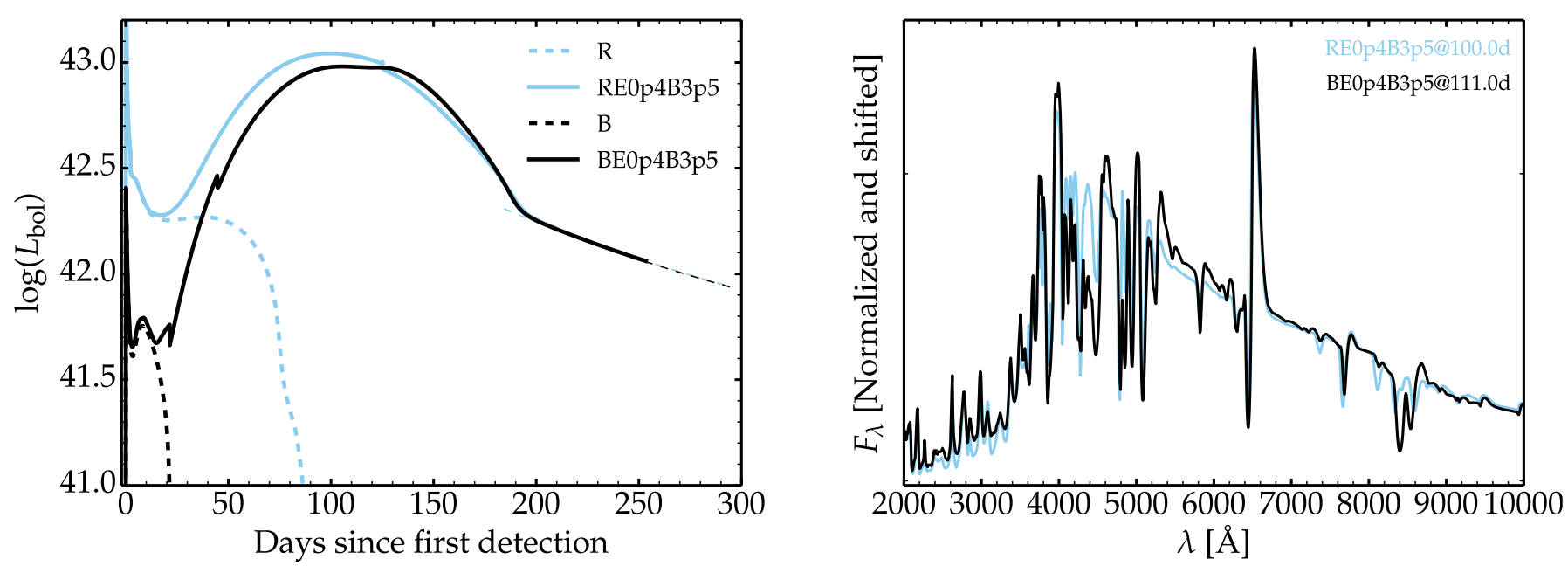

Fig. 4. Left: bolometric light curve computed with HERACLES for models RE0p4B3p5 (RSG progenitor) and BE0p4B3p5 (BSG progenitor) under the influence of a magnetar (thick line) or not (thick dashed line). The thin dashed line corresponds to the magnetar power at $>250 \mathrm{~d}$. Prior to the influence of magnetar power on the emergent light, the luminosity stems primarily from shock deposited energy. It is at such early times that one may distinguish a BSG from a RSG star as the progenitor of a super-luminous SN. Right: maximum light spectra (around $100 \mathrm{~d}$ after explosion) for the two models shown at left.

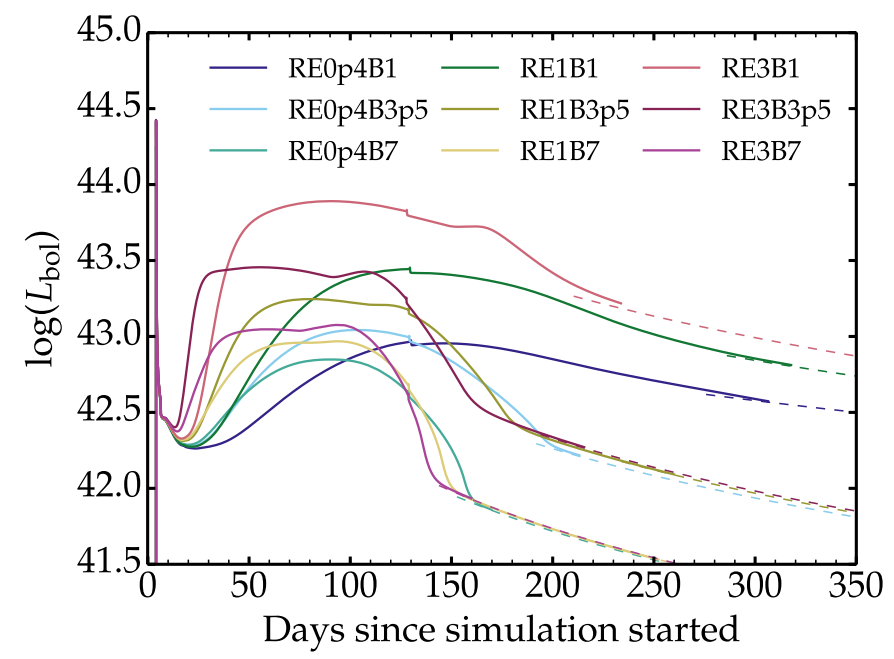

Fig. 5. Bolometric light curves for the grid of magnetar-powered SN models based on the RSG progenitor. The glitch at $125 \mathrm{~d}$ occurs when the outer shock with the progenitor wind leaves the Eulerian grid at $10^{16} \mathrm{~cm}$. In Appendix A, we present light curves for model sets sharing the same $E_{\mathrm{pm}}$ or the same $B_{\mathrm{pm}}$ (see Sect. 6 for discussion).

$t_{\text {peak }}$ and $L_{\text {peak }}$ are broadly consistent ${ }^{2}$ with the values obtained from the analytic expressions (15) and (16) in Kasen \& Bildsten (2010). Beyond maximum, the bolometric luminosity falls onto the instantaneous magnetar luminosity (which then scales as the inverse of $\left.B_{\mathrm{pm}}^{2}\right)$ after a time similar to $t_{\text {peak }}$.

\subsection{Energetics}

Figure 6 illustrates the energetics in our simulation RE1B3p5. In the top panel, we show the evolution of the total gas energy $E_{\text {kin+th }}$ (kinetic plus thermal contributions), the trapped radiation energy $E_{\text {rad,trapped }}$, the cumulative energy deposited by the magnetar $E_{\text {dep,pm }}$, and the cumulative radiative losses through the outer boundary $E_{\text {rad,losses }}$. We also plot the quantity $\Delta E \equiv$

2 The broad and flat light curve maxima can complicate an accurate estimate of the time of maximum.
$E_{\text {kin+th }}+E_{\text {rad,trapped }}+E_{\text {rad,losses }}-E_{\text {dep,pm }}$, which should be constant. This illustration shows that initially (the model is within hours of shock breakout) the trapped radiation energy is rapidly turned into kinetic energy while the supply of energy from the magnetar boosts both the trapped radiation energy and the ejecta kinetic energy. At 120 d, a little more than $10^{50}$ erg has been radiated to infinity, which is about ten times what this model would radiate in the absence of a magnetar (assuming that a representative Type II SN, not influenced by a central source, radiates $10^{49}$ erg over its entire lifetime). In this model RE1B3p5, the bulk of the magnetar energy has been channeled into ejecta kinetic energy, but yielding only a $60 \%$ increase in ejecta kinetic energy (not the factor of ten above for the radiative energy losses) over the value it would have had in the absence of a magnetar (at $120 \mathrm{~d}$ the magnetar in this model still has about $2 \times 10^{50} \mathrm{erg}$ to radiate).

The middle and bottom panels in Fig. 6 illustrate how efficiently the magnetar energy is channelled into escaping radiation (referred to as $E_{\text {rad,losses }}$ ). Nearly $10^{51}$ erg is radiated away in model RE3B1, while the rest of the models falls in the range $0.05-0.3 \times 10^{51} \mathrm{erg}$. The luminosity boost over a standard SN II therefore goes from minor $(5 x)$ to very large $(100 \times)$. When normalized to the current cumulative energy deposited by the magnetar, the most efficient "engines" are the magnetars with long spin down time scales, that is, those with a lower magnetic field and/or a lower initial rotation energy (longer periods). These trends and quantities would not be significantly altered if we had started the magnetar energy deposition at the magnetar birth because it is only radiation emitted on a long time scale that boosts the bolometric luminosity of the SN (Kasen \& Bildsten 2010).

\subsection{Photospheric properties}

We show the evolution of the photospheric properties for our magnetar-powered SN models arising from the explosion of a RSG progenitor in Fig. 7. Compared to the model without magnetar (dashed line), the photospheric velocity is at all times larger, and by a large factor after $100 \mathrm{~d}$ since the no-magnetar model is by then optically thin. The effect illustrated here does not arise from a change in ejecta kinetic energy (which is essentially fixed after 50-100 d; see Fig. 6 and Appendix A) but stems 

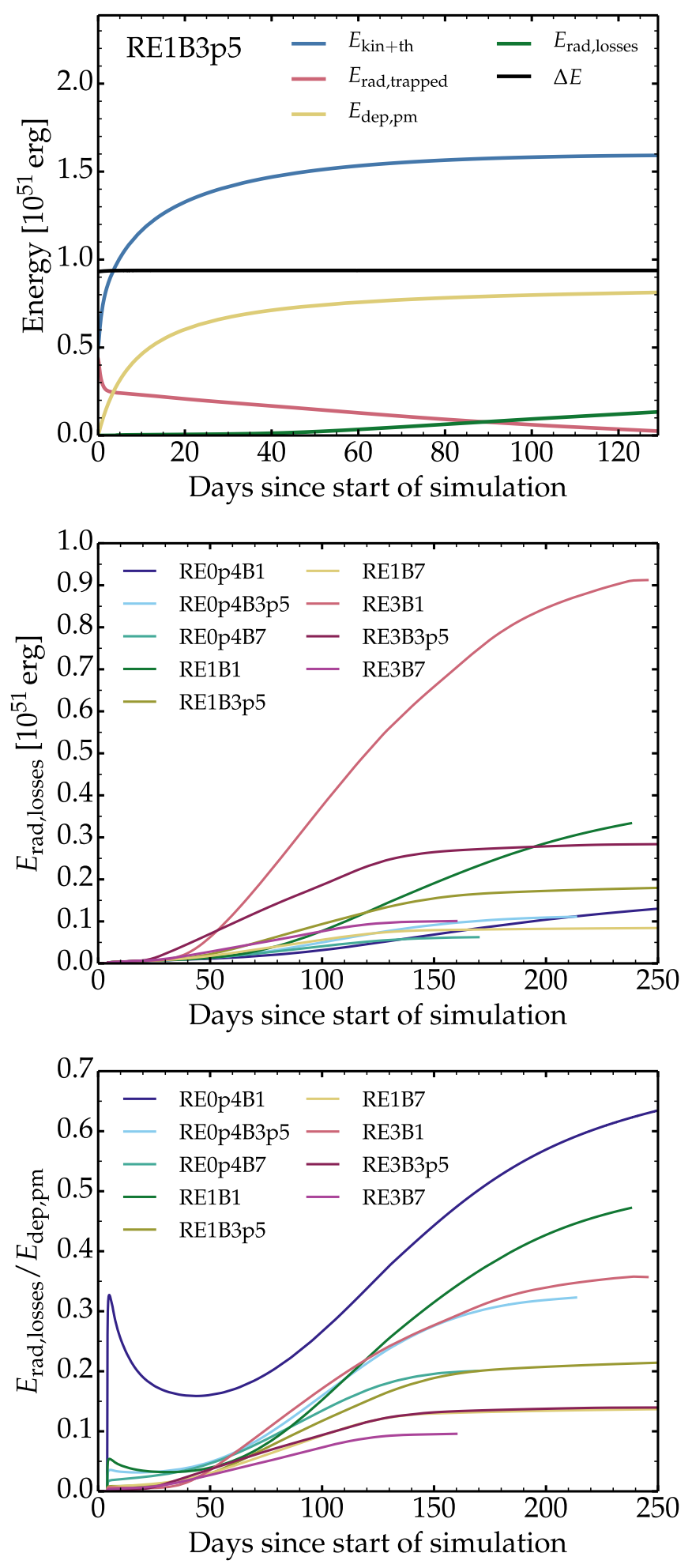

Fig. 6. Top: evolution of the various energy components in the RE1B3p5 simulation. We show $E_{\text {kin th }}$, which sums the kinetic and thermal (i.e., gas) energy; $E_{\text {rad,trapped }}$, which is the trapped radiation energy; $E_{\mathrm{dep}, \mathrm{pm}}$, which is the energy deposited by the magnetar; $E_{\text {rad,losses }}$, which is the radiative energy streaming out through the outer grid boundary; and $\Delta E \equiv E_{\text {kin }+ \text { th }}+E_{\text {rad,trapped }}+E_{\text {rad,losses }}-E_{\text {dep,pm }}$, which tracks conservation of energy. The start of the simulation is $1.15 \mathrm{~d}$ in all simulations - any magnetar energy deposited prior to that is ignored. Middle and bottom: evolution of the radiative losses (middle) and of the fraction of the magnetar deposited energy that escapes as radiation as a function of time (bottom) for the magnetar-powered SN models based on the RSG progenitor. Additional illustrations are provided in Appendix A. instead from the higher ionization of the ejecta. In the presence of a magnetar, the recombination is inhibited or delayed. The near plateau in the photospheric velocity after 50-100 d implies that the photosphere resides in a narrow range of ejecta mass shells (until $>250 \mathrm{~d}$ in some models).

The photospheric temperature is larger at all times in the presence of a magnetar. ${ }^{3}$ In many models, this temperature is close to the recombination temperature of hydrogen, which implies that the boost to the model luminosity is caused by the greater photospheric radius.

Interestingly, in the models with $E_{\mathrm{pm}}>10^{51} \mathrm{erg}$, the initial decline of the photospheric temperature is halted at $10-40 \mathrm{~d}$ after the magnetar birth and the temperature starts rising again. In the models with the faster rotating magnetars (RE3B1 and RE3B3p5), the photospheric temperature reaches a maximum of about $10000 \mathrm{~K}$, which is sufficient to re-ionize hydrogen (in model RE3B7, the spin-down time scale is so short that most of the energy goes into work to accelerate the ejecta and the boost to $T_{\text {phot }}$ is smaller than for models RE3B1 and RE3B3p5).

A non-monotonic evolution of the photospheric temperature and of the location at maximum absorption in He I $5875 \AA$ have been observed in the Type Ib SN 2005bf (Folatelli et al. 2006). This is consistent with the magnetar influence invoked to reproduce the light curve of SN 2005bf (Maeda et al. 2007).

\subsection{Spectral properties at bolometric maximum}

We conclude this section by discussing the optical spectra for our grid of models based on the RSG progenitor (Fig. 8). This posttreatment of non-monotonic ejecta with CMFGEN is currently only possible during the photospheric phase so we choose the unambiguous time of bolometric maximum for this illustration.

The maximum light spectra shown in Fig. 8 cover a range of optical colors, reflecting the range in photospheric temperature (Fig. 7). Bluer spectra appear like standard Type II SNe at early times, when the conditions at the photosphere are ionized or partially ionized. In model RE3B 1 at $100 \mathrm{~d}$, we recognize lines of H I, He I, and N II, as in the earliest spectra for SN 1999em (Dessart \& Hillier 2005, 2006). Redder spectra appear like the standard Type II SNe at later times in the photospheric phase, when the conditions at the photosphere are partially neutral (and an $\mathrm{H}$ recombination front has formed; Dessart \& Hillier 2011).

The line widths are, however, anomalously low for the SN luminosity. Indeed, the photospheric velocities at bolometric maximum are about $4000 \mathrm{~km} \mathrm{~s}^{-1}$ (Fig. 7 and Table 1), which is standard for a Type II (Hamuy 2003), but the luminosity is up to 10-50 times larger than standard. In our choice of magnetar properties, the energy released by the magnetar boosts primarily the radiation budget and affects little the kinetic energy (choosing a magnetar with a larger rotational energy and larger magnetic field would do the opposite). In doing so, it breaks the tight correlation between brightness and expansion rate inferred from the observation of Type II SNe (Hamuy 2003).

\footnotetext{
3 This holds except for model RE0p4B1, which is slightly cooler at the photosphere. The energy released by the magnetar does not in principle need to produce a higher photospheric temperature compared to the same model without a magnetar. Indeed, the influence of the magnetar may simply be to push the photosphere outward in mass/velocity space, while the photospheric temperature remains close the recombination temperature of hydrogen. In a SN II, the photosphere at the recombination epoch coincides with the layer where hydrogen changes ionization, from neutral to ionized. As long as there is such a recombination front, the photosphere is at roughly the same temperature, around $6000 \mathrm{~K}$.
} 

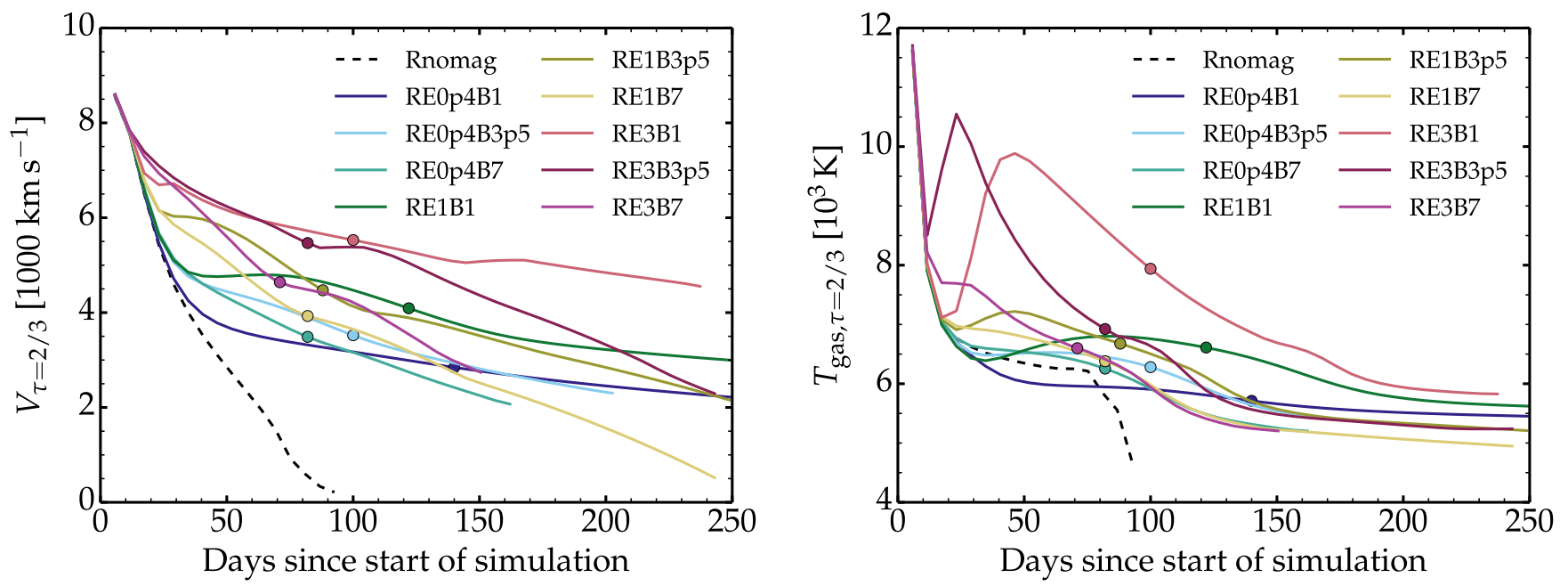

Fig. 7. Evolution of the photospheric velocity with time in the HERACLES simulations for the RSG explosion model powered by a variety of magnetar energy and field strength. The colored dot on each curve corresponds to the time at which we compute the spectra shown in Fig. 8.

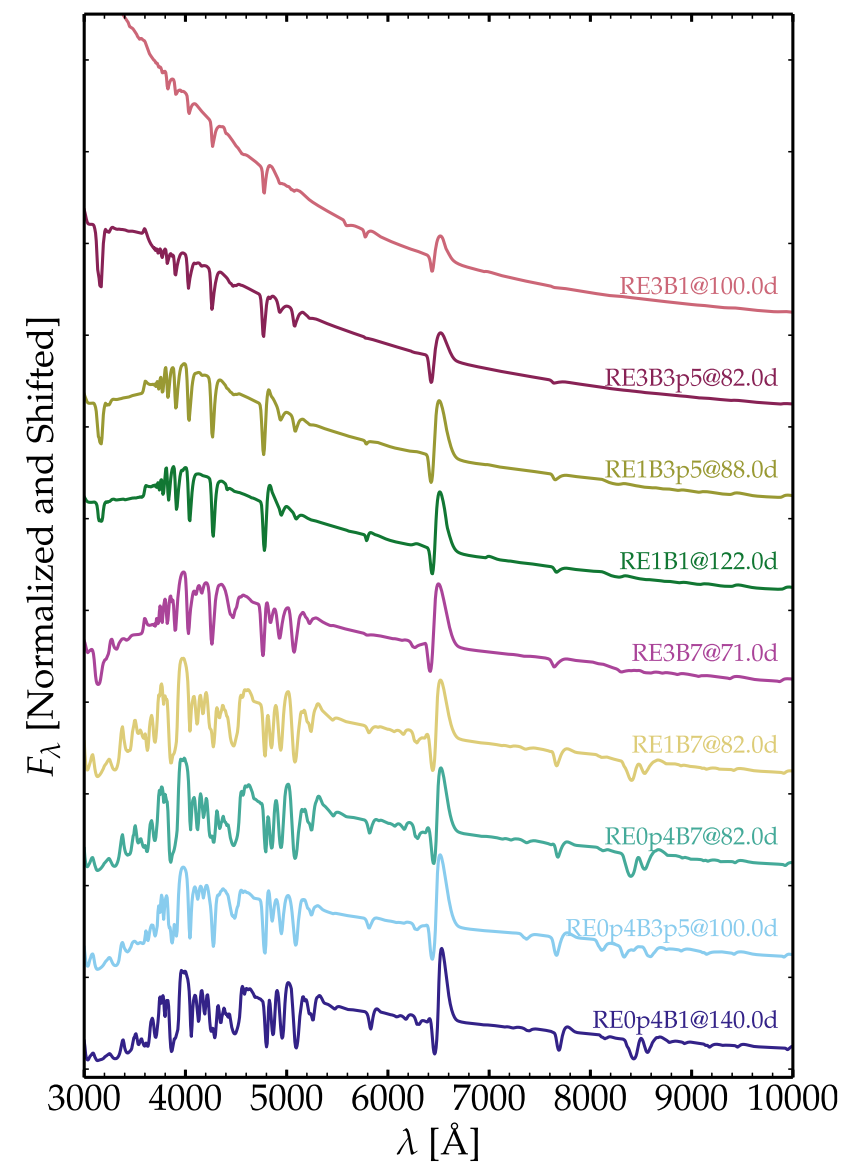

Fig. 8. Model spectra computed with CMFGEN for the magnetarpowered RSG explosion models and corresponding to an epoch around bolometric maximum (see label for model name and post-explosion epoch). We stack the spectra vertically starting with the reddest at the bottom (model Re0p4B1) and progressing upward toward bluer spectra. Each flux spectrum is normalized at $10000 \AA$ and shifted vertically for visibility (the ordinate tick mark gives the zero-flux level).

Some observed Type II SNe, with no apparent sign of interaction, appear over-luminous for their expansion rate, for example, LSQ13fn (Polshaw et al. 2016) or SN 2006V (Taddia et al. 2012), and this may be a signature of a magnetar influence.

\section{Comparison to observations}

Few observations of super-luminous Type II SNe suggest a magnetar influence. Kasen \& Bildsten (2010) propose a magnetar with $B_{\mathrm{pm}}=2 \times 10^{14} \mathrm{G}, E_{\mathrm{pm}}=5 \times 10^{51} \mathrm{erg}$ (rotation period of $2 \mathrm{~ms}$ ), and a H-rich ejecta of $5 M_{\odot}$ to explain the light curve of SN 2008es (Gezari et al. 2009; Miller et al. 2009). Such a low mass ejecta is hard to fit within the context of an H-rich massive star progenitor so we leave this object aside for now.

Here, we choose a case that seems to be less ambiguous. We compare our simulations to the super-luminous Type II SN named OGLE-SN14-073 (Terreran et al. 2017), which exhibits a bolometric light curve similar to that of SN 1987A but brighter by a factor of ten and significantly broader (Fig. 9, big black circles) - the time integrated luminosity up to $>200 \mathrm{~d}$ is about $10^{50} \mathrm{erg}$. The photospheric velocity inferred during the high brightness phase is about $6000 \mathrm{~km} \mathrm{~s}^{-1}$. Terreran et al. (2017) propose a RSG progenitor with an explosion model yielding a very large ejecta mass of about $60 M_{\odot}$ and kinetic energy of $12 \times 10^{51} \mathrm{erg}$. Their quoted uncertainties are however quite large.

We have compared our grid of models and found that RE0p4B3p5 and RE0p4B7 match closely the bolometric light curve. These models arise from an ejecta of $11.9 M_{\odot}$ and a kinetic energy of about $10^{51} \mathrm{erg}$, so 10 times less energetic and 5 times less massive than proposed for OGLE-SN14-073 . Because their luminosity is too large at late times and too small at early times, we performed a new simulation (using the same initial ejecta model) with a higher magnetar field $\left(B_{\mathrm{pm}}=4.5 \times 10^{14} \mathrm{G}\right)$ and a broader energy deposition profile (in order to hasten the magnetar influence and produce a higher luminosity at early times the alternative of a greater progenitor radius would also yield a greater brightness at early times).

In Fig. 9, we compare the bolometric light curve of this new model RE0p4B4p5o with the observations of OGLE-SN14-073, and include SN 1987A for reference. Model RE0p4B4p5o matches quite closely OGLE-SN14-073. However, the photospheric velocity of these three models is about $4000 \mathrm{~km} \mathrm{~s}^{-1}$ at maximum, which is $50 \%$ lower than inferred for OGLE-SN14-073. Scaling the initial density and velocity by $50 \%$, as well as scaling the temperature by $22 \%$ (the model is pre-breakout hence half the total shock deposited energy is radiation, the other half is kinetic) we produce a new model RE0p4B4p5os (total mass of $17.8 M_{\odot}$ and a kinetic energy of 


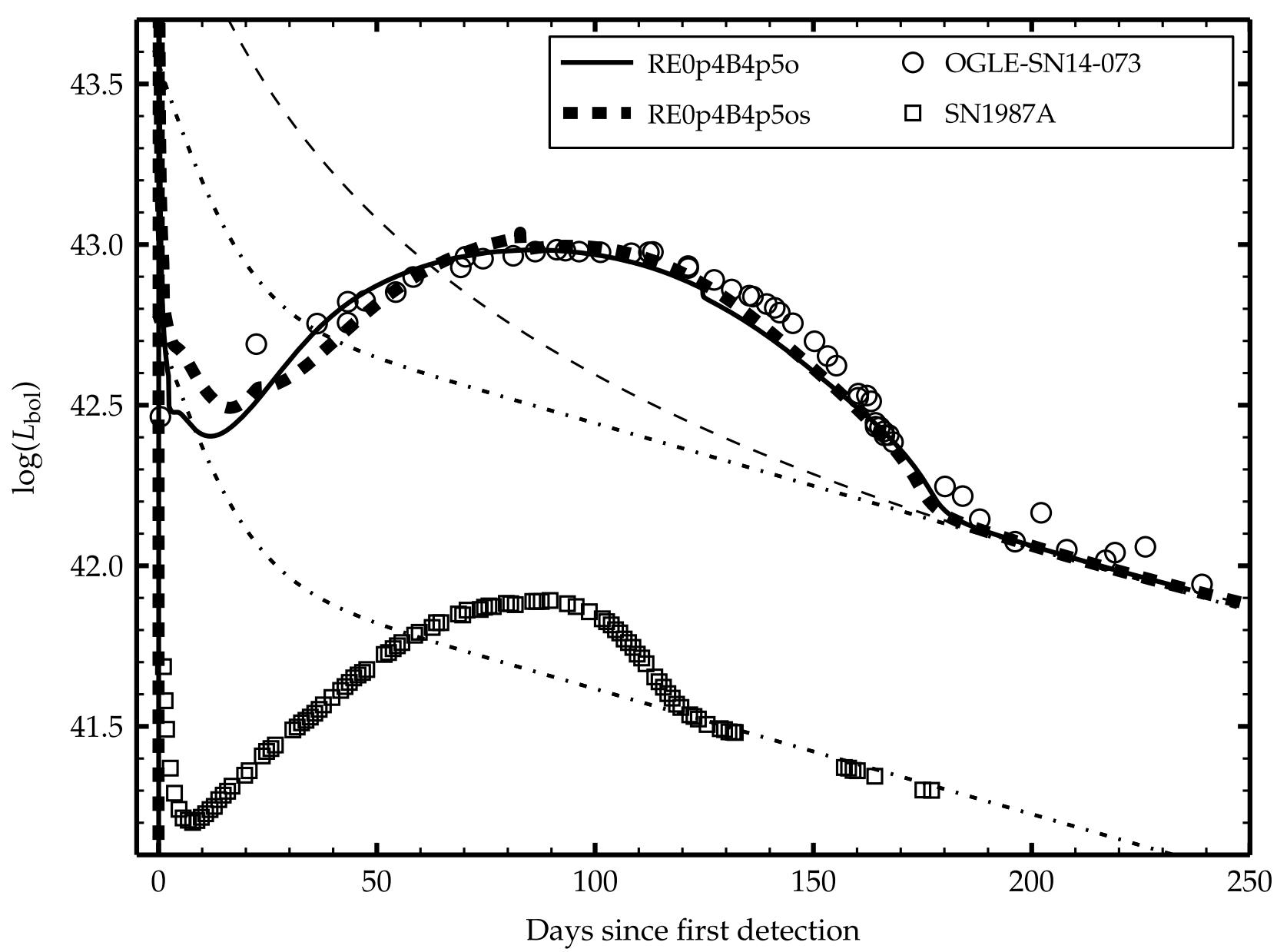

Fig. 9. Comparison of the bolometric light curves of magnetar-powered models RE0p4B4p5o (thick dashed line) and RE0p4B4p5os (thin line) with that inferred for OGLE-SN14-073 (empty circles; Terreran et al. 2017) and SN 1987A (empty squares; Hamuy et al. 1988). The origin of the $x$-axis is the time of the first signal detection at the outer grid boundary for the models ( $\sim 4 \mathrm{~d}$ after the start of the simulations), or the inferred time of explosion for the observations. We add the magnetar power of these two models (thin dashed line) and the radioactive decay power from $0.07 M_{\odot}$ of ${ }^{56} \mathrm{Ni}$ (to match the nebular luminosity of SN 1987A; dash-dotted line). To power the nebular luminosity of OGLE-SN14-073 with radioactive decay requires $0.47 M_{\odot}$ of ${ }^{56} \mathrm{Ni}$ (upper dash-dotted line).

$\left.2.67 \times 10^{51} \mathrm{erg}\right)$. This choice of scaling is to increase the velocity while maintaining the same diffusion time (which scales as $\sqrt{M / V}$ ). RE0p4B4p5os yields a similar light curve to OGLESN14-073. However, the photospheric velocity at bolometric maximum is only increased to $4280 \mathrm{~km} \mathrm{~s}^{-1}$, hence still significantly lower than the inferred value of $6000 \mathrm{~km} \mathrm{~s}^{-1}$ for OGLESN14-073. Perhaps the kinetic energy is not underestimated and there is instead an error in inferring the photospheric velocity. The magnetar influence may affect line profiles such that the location of maximum line absorption occurs at a Doppler velocity significantly greater than the photospheric velocity value we report (which accounts only for electron scattering). Time dependence might also affect line profiles by preventing the formation of a steep recombination front as normally seen in standard Type II SNe (Utrobin \& Chugai 2005; Dessart \& Hillier 2008). Numerical explorations of magnetar-powered Type II SNe with CMFGEN show that spectral lines can remain very broad until late times, and broader than predicted using steady state (Dessart, in prep.). This aspect requires further study. But it seems likely that OGLE-SN14-073 is a unique Type II event, combining a massive and very energetic ejecta with a magnetar having $B_{\mathrm{pm}}=4.5 \times 10^{14} \mathrm{G}$ and $E_{\mathrm{pm}}=0.4 \times 10^{51} \mathrm{erg}$.

The ejecta corresponding to RE0p4B4p5os has a kinetic energy and mass greater than standard for a Type II SN but less extreme than proposed by Terreran et al. (2017). Although not explicitly stated in their paper, the model of Terreran et al. requires $0.47 M_{\odot}$ of ${ }^{56} \mathrm{Ni}$, while in our magnetar powered model we assume no ${ }^{56} \mathrm{Ni}$. The influence on the bolometric light should be similar since the power released in each process is similar (see upper dash-dotted and dashed lines in Fig. 9). In the radioactive decay scenario, this ${ }^{56} \mathrm{Ni}$ mass makes sense since the total decay energy from $1 M_{\odot}$ of ${ }^{56} \mathrm{Ni}$ is $\sim 2 \times 10^{50} \mathrm{erg}$, and the time integrated luminosity of OGLE-SN14-073 is about $10^{50} \mathrm{erg}$. The difference between a magnetar-powered model and a ${ }^{56} \mathrm{Ni}$ powered model is however non trivial when considering spectra and colors (Dessart et al. 2012). In OGLE-SN14-073, the presence of a Type II spectrum at all times with little evidence for metal lines (Terreran et al. 2017) is hard to combine with the presence of a large progenitor $\mathrm{CO}$ core required to yield a large ${ }^{56} \mathrm{Ni}$ mass. In explosion models powered by ${ }^{56} \mathrm{Ni}$, the spectrum formation region at the time of bolometric maximum is located in metal rich regions (Dessart et al. 2013b), which is in conflict with the observations of OGLE-SN14-073. If the power source is a magnetar, the ejecta composition may instead be dominated by $\mathrm{H}$ and $\mathrm{He}$, which is compatible with the Type II spectrum observed at all times in OGLE-SN14-073.

At the time of bolometric maximum, the luminosity is a factor of 2 above the concomitant magnetar power in models 
RE0p4B4p5o and RE0p4B4p5os - similar offsets are present in the set of simulations shown in Fig. 5. An 80\% offset is seen for SN 1987A between the bolometric maximum and the concomitant decay power from $0.07 M_{\odot}$ of ${ }^{56} \mathrm{Ni}$. Arnett rule (Arnett 1982) states that the peak bolometric luminosity should be equal to the power from which it derives (magnetar radiation, radioactive decay).

\section{Conclusion}

We have presented 1D gray radiation-hydrodynamics simulations of magnetar-powered Type II SNe performed with the code HER ACLES (González et al. 2007; Vaytet et al. 2011). Our simulations are based on BSG and RSG progenitors, which reproduce roughly the properties of SN 1987A (Dessart \& Hillier 2010) and SN 1999em (Dessart et al. 2013a) if ${ }^{56} \mathrm{Ni}$ decay power (rather than magnetar power) is accounted for. We adopt a power source from a magnetic dipole for convenience. In practice, the power radiated by such a compact remnant may not follow such a smooth evolution.

In our supergiant progenitors, the composition is dominated by $\mathrm{H}$ and $\mathrm{He}-$ the contribution in metals from the He core is secondary. In our magnetar-powered SN simulations, allowing for chemical stratification using five representative species $(\mathrm{H}$, $\mathrm{He}, \mathrm{O}, \mathrm{Si}$, and ${ }^{56} \mathrm{Ni}$ ) makes little difference compared to assuming a composition typical of a RSG H-rich envelope. Nearly all our simulations therefore assume a uniform $\mathrm{H}$-rich composition. Furthermore, we mimic multi-D fluid instabilities seen in the simulations of Chen et al. (2016); Suzuki \& Maeda (2017) by depositing the magnetar energy over a range of masses, rather than over a narrow mass range at the base. With this approach, the density and temperature structures remain smooth at all times, the light curves rise earlier and do not show a late time bump in the photospheric phase.

We find that early-time observations may help constrain the size of the progenitor star. Provided the magnetar influence does not start too soon, the SN luminosity arising from a BSG explosion is markedly lower than that from a RSG explosion. By the time of maximum, the influence of the progenitor radius on expansion cooling is swamped by the large energy release from the magnetar. The light curves from the BSG and RSG magnetarpowered $\mathrm{SNe}$ overlap at and beyond maximum. Similarly, the optical spectra at maximum are similar and one would not be able to discern between the two progenitors from such a spectral information.

We then present a grid of models for RSG explosions influenced by a magnetar. For our set of magnetar field $\left(1.0-7.0 \times 10^{14} \mathrm{G}\right)$ and magnetar initial rotational energy $\left(0.4-3.0 \times 10^{51} \mathrm{erg}\right)$, the resulting bolometric light curves reach a broad peak at 50-150d after explosion with a power of $0.7-7.8 \times 10^{43} \mathrm{erg} \mathrm{s}^{-1}$. The fraction of the magnetar energy channelled into (UV-optical-infrared) $\mathrm{SN}$ radiation is higher for weaker field but in all cases the boost to the SN luminosity is significant (between a factor of 5 and 100 compared to a standard Type II SN). The magnetar influence delays the recombination of the ejecta and maintains the photosphere at a larger radius (in a mass shell located further out in the ejecta). In some cases, the magnetar energy deposition reverses the cooling and causes the photosphere to heat up after a few months. SN 2005bf exhibits a similar phenomenon (Folatelli et al. 2006), which is compatible with a magnetar origin (Maeda et al. 2007). The maximum light spectra are analogous to those of standard SNe II, with a range in colors that reflects the scatter in photospheric temperature (itself dependent on the heating efficiency of the magnetar). However, the modest expansion rate and huge brightness of these magnetar powered $\mathrm{SNe}$ breaks the brightness/expansion-rate correlation observed in standard SNe II (Hamuy 2003).

Amongst super-luminous SNe II, it appears that OGLESN14-073 (Terreran et al. 2017) may be interpreted as a magnetar powered SN. We find that our RSG model influenced by a magnetar with $E_{\mathrm{pm}}=0.4 \times 10^{51} \mathrm{erg}$ and $E_{\mathrm{pm}}=4.5 \times 10^{14} \mathrm{G}$ yields a satisfactory match to the bolometric light curve, although our model underestimates the inferred expansion rate.

The main uncertainties in our simulations are the very approximate handling of the impact of multi-dimensional fluid instabilities (which will introduce clumping; Jerkstrand et al. 2017), the neglect of time dependence in the non-LTE solution, and the neglect of non-thermal effects associated with the energy injection from the magnetar. Further modeling is therefore needed, for example to test whether the line profile widths are broadened by the magnetar influence and time dependent effects, which could potentially lead to overestimating the ejecta kinetic energy and mass.

Acknowledgements. We thank Giacomo Terrreran for providing the estimated bolometric luminosity of OGLE-SN14-073. LD thanks ESO-Vitacura for their hospitality. This work utilized computing resources of the mesocentre SIGAMM, hosted by the Observatoire de la Côte d'Azur, Nice, France. This research was supported by the Munich Institute for Astro- and Particle Physics (MIAPP) of the DFG cluster of excellence "Origin and Structure of the Universe".

\section{References}

Arnett, W. D. 1982, ApJ, 253, 785

Barkat, Z., Rakavy, G., \& Sack, N. 1967, Phys. Rev. Lett., 18, 379

Bersten, M. C., Benvenuto, O. G., Orellana, M., \& Nomoto, K. 2016, ApJ, 817, L8

Bucciantini, N., Quataert, E., Arons, J., Metzger, B. D., \& Thompson, T. A. 2008, MNRAS, 383, L25

Bucciantini, N., Quataert, E., Metzger, B. D., et al. 2009, MNRAS, 396, 2038 Burrows, A., Dessart, L., Livne, E., Ott, C. D., \& Murphy, J. 2007, ApJ, 664, 416 Chen, K.-J., Woosley, S. E., \& Sukhbold, T. 2016, ApJ, 832, 73

Chevalier, R. A., \& Dwarkadas, V. V. 1995, ApJ, 452, L45

Dessart, L., \& Hillier, D. J. 2005, A\&A, 437, 667

Dessart, L., \& Hillier, D. J. 2006, A\&A, 447, 691

Dessart, L., \& Hillier, D. J. 2008, MNRAS, 383, 57

Dessart, L., \& Hillier, D. J. 2010, MNRAS, 405, 2141

Dessart, L., \& Hillier, D. J. 2011, MNRAS, 410, 1739

Dessart, L., Hillier, D. J., Waldman, R., Livne, E., \& Blondin, S. 2012, MNRAS, 426, L76

Dessart, L., Hillier, D. J., Waldman, R., \& Livne, E. 2013a, MNRAS, 433, 1745 Dessart, L., Waldman, R., Livne, E., Hillier, D. J., \& Blondin, S. 2013b, MNRAS, 428, 3227

Dessart, L., Audit, E., \& Hillier, D. J. 2015, MNRAS, 449, 4304

Dessart, L., Hillier, D. J., Audit, E., Livne, E., \& Waldman, R. 2016, MNRAS, 458, 2094

Duncan, R. C., \& Thompson, C. 1992, ApJ, 392, L9

Folatelli, G., Contreras, C., Phillips, M. M., et al. 2006, ApJ, 641, 1039

Gal-Yam, A., Mazzali, P., Ofek, E. O., et al. 2009, Nature, 462, 624

Gezari, S., Halpern, J. P., Grupe, D., et al. 2009, ApJ, 690, 1313

González, M., Audit, E., \& Huynh, P. 2007, A\&A, 464, 429

Gutiérrez, C. P., Anderson, J. P., Hamuy, M., et al. 2017, ApJ, 850, 89

Hamuy, M. 2003, ApJ, 582, 905

Hamuy, M., Suntzeff, N. B., Gonzalez, R., \& Martin, G. 1988, AJ, 95, 63

Inserra, C., Smartt, S. J., Jerkstrand, A., et al. 2013, ApJ, 770, 128

Jerkstrand, A., Smartt, S. J., Inserra, C., et al. 2017, ApJ, 835, 13

Kasen, D., \& Bildsten, L. 2010, ApJ, 717, 245

Maeda, K., Tanaka, M., Nomoto, K., et al. 2007, ApJ, 666, 1069

Metzger, B. D., Giannios, D., Thompson, T. A., Bucciantini, N., \& Quataert, E. 2011, MNRAS, 413, 2031

Metzger, B. D., Margalit, B., Kasen, D., \& Quataert, E. 2015, MNRAS, 454, 3311

Miller, A. A., Chornock, R., Perley, D. A., et al. 2009, ApJ, 690, 1303

Mösta, P., Ott, C. D., Radice, D., et al. 2015, Nature, 528, 376

Nicholl, M., Smartt, S. J., Jerkstrand, A., et al. 2013, Nature, 502, 346

Nicholl, M., Smartt, S. J., Jerkstrand, A., et al. 2014, MNRAS, 444, 2096 
A\&A 613, A5 (2018)

Ofek, E. O., Cameron, P. B., Kasliwal, M. M., et al. 2007, ApJ, 659, L13

Polshaw, J., Kotak, R., Dessart, L., et al. 2016, A\&A, 588, A1

Smith, N., Li, W., Foley, R. J., et al. 2007, ApJ, 666, 1116

Stoll, R., Prieto, J. L., Stanek, K. Z., et al. 2011, ApJ, 730, 34

Sukhbold, T., \& Woosley, S. E. 2016, ApJ, 820, L38

Suzuki, A., \& Maeda, K. 2017, MNRAS, 466, 2633

Taddia, F., Stritzinger, M. D., Sollerman, J., et al. 2012, A\&A, 537, A140

Taddia, F., Sollerman, J., Fremling, C., et al. 2018, A\&A, 609, A106
Terreran, G., Pumo, M. L., Chen, T.-W., et al. 2017, Nat. Astron., 1, 228

Thompson, T. A., Chang, P., \& Quataert, E. 2004, ApJ, 611, 380

Usov, V. V. 1992, Nature, 357, 472

Utrobin, V. P., \& Chugai, N. N. 2005, A\&A, 441, 271

Utrobin, V. P., Wongwathanarat, A., Janka, H.-T., \& Müller, E. 2017, ApJ, 846, 37

Vaytet, N. M. H., Audit, E., Dubroca, B., \& Delahaye, F. 2011, J. Quant. Spectr. Rad. Transf., 112, 1323

Woosley, S. E. 2010, ApJ, 719, L204 


\section{Appendix A: Additional illustrations}
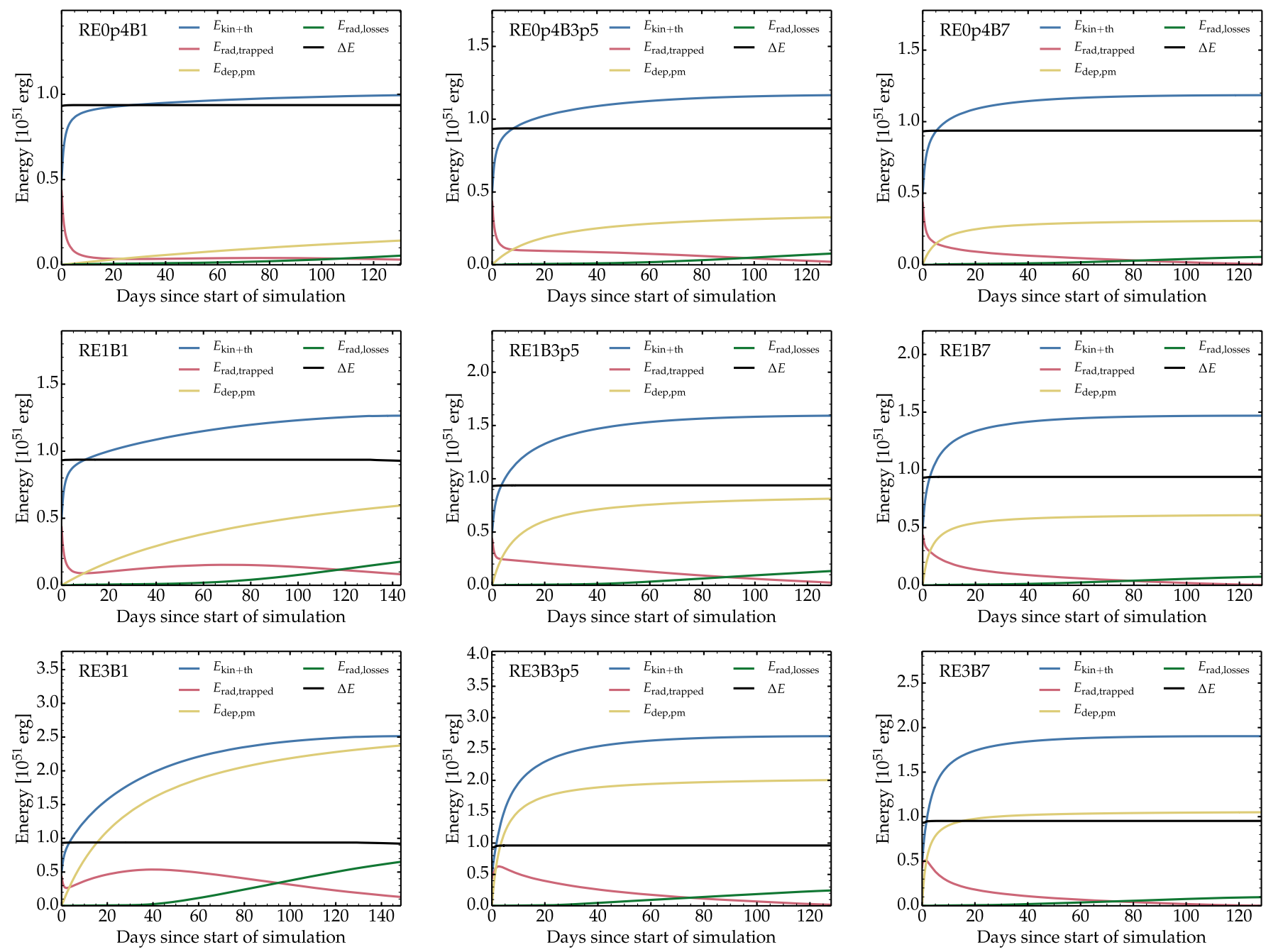

Fig. A.1. Evolution of the various energy components in the HER ACLES simulations. We show $E_{\text {kintth }}$, which sums the kinetic and thermal (i.e., gas) energy; $E_{\text {rad,trapped }}$, which is the trapped radiation energy; $E_{\text {dep.pm }}$, which is the energy deposited by the magnetar; $E_{\text {rad,losses }}$, which is the radiative energy streaming out through the outer grid boundary; and $\Delta E$, which is defined as $E_{\text {kin+th }}+E_{\text {rad,trapped }}+E_{\text {rad,losses }}-E_{\text {dep,pm }}$ and should be constant. The start of the simulation is $1.15 \mathrm{~d}$ in all simulations - any magnetar energy deposited prior to that is not accounted for in the HERACLES simulations.

In this appendix we provide additional illustrations for the models discussed in the main body of the paper. In Fig. A.1, we discuss the evolution of the various forms of energy on the grid, similarly to the top panel in Fig. 6.

We also show in Fig. A.2 the bolometric light curves for the magnetar-powered SN models discussed in Sect. 6 but this time grouped by triads of the same magnetar initial rotational energy (but different magnetic field) or the same magnetic field (but different magnetar rotational energy). 

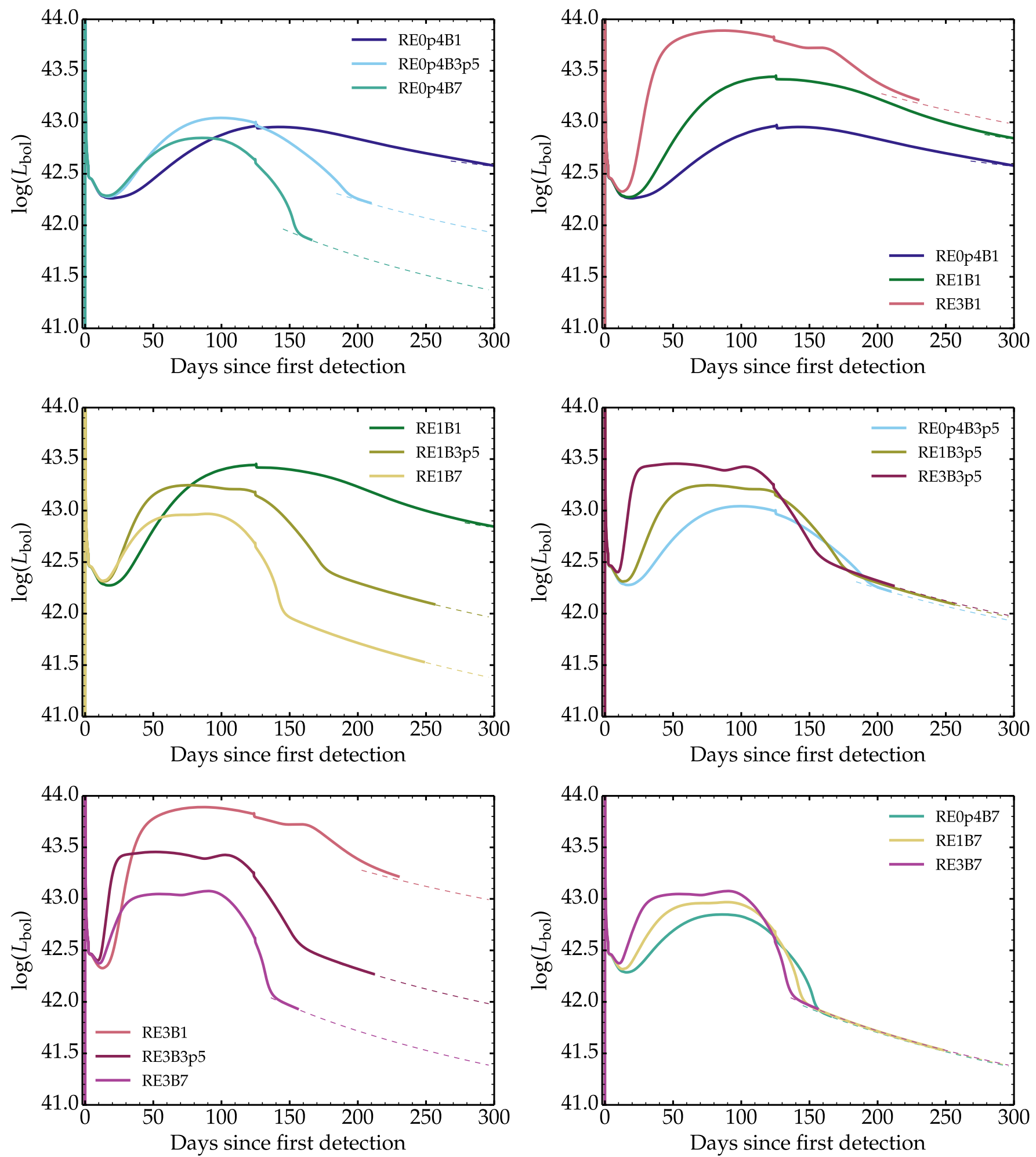

Fig. A.2. Bolometric light curves from HERACLES simulations for the RSG progenitor. At $\sim 125 \mathrm{~d}$, the shock between ejecta and progenitor wind leaves the grid and causes a slight glitch in the luminosity. In some models (e.g., RE3B7), the light curve shows a small bump at late times in the photospheric phase. This feature is associated with the dense shell that results from the dynamical influence of the magnetar power (even though we adopt a broad energy deposition profile; see Sect. 3). Models with a higher initial magnetar energy and/or a higher magnetar field tend to produce a more massive dense shell and show this late light curve bump. 